\title{
Mensuração do Capital Social Acumulado a partir de Interações Sociais em Páginas Institucionais no Facebook
}

\author{
Kaique Matheus R. Cunha ${ }^{1}$, Alan Keller Gomes ${ }^{1}$ \\ ${ }^{1}$ Instituto Federal de Educação, Ciência e Tecnologia de Goiás (IFG) - Câmpus Inhumas \\ Av. Universitária, s/nº , Vale das Goiabeiras, Inhumas-GO - Brasil - CEP: 75400-000 \\ ifgkaique@gmail.com, alan.gomes@ifg.edu.br
}

\begin{abstract}
Within Online Social Networks, functionalities which allow the interaction among users are setting up network sociability practices. Actions such as Post, Comment, Like, Share, have a special meaning when focused by Digital Sociology. With the support of Pierre Bourdieu's theory, we identify and capture data related to sociability practices within Facebook Institutional Fanpages. Then, we use such data in actions sequence learning. By measuring the frequence of occurence of the learned sequences, we build an indicator of the accumulated Social Capital. From such indicator and the number of captured social interactions, we compute the accumulated Social Capital volume.

Resumo. Funcionalidades que permitem a interação entre usuários dentro das Redes Sociais Online configuram práticas de sociabilidade em rede, ou seja, ações como Publicar, Comentar, Curtir e Compartilhar têm um significado especial quando estão sob o foco da Sociologia Digital. Com o apoio da teoria de Pierre Bourdieu, neste trabalho são identificados e capturados dados relacionados às práticas de sociabilidade em rede dentro de Páginas Institucionais no Facebook. Esses dados são utilizados no aprendizado da sequência de ações. A partir da frequência de ocorrências das seqüências aprendidas, um indicador do Capital Social acumulado é apresentado. A partir desse indicador e do número de interações sociais, o volume de Capital Social é computado.
\end{abstract}

\section{Introdução}

Em uma sociedade cada vez mais conectada e mediada por tecnologias interativas, as Redes Sociais Online (RSO) tem promovido mudanças sociais profundas, a tal ponto, que essas redes digitais se fundem e estendem as redes sociais da vida real. As RSO são facilitadores tecnológicos que contribuem para o fortalecimento de laços sociais da vida real e para o estabelecimento de novos laços [Santos e Cypriano 2014]. Além disso, elas incorporam em suas funcionalidades o caráter lúdico e simétrico que define a sociabilidade nas redes sociais do mundo real [Santos e Cypriano 2011].

Funcionalidades como Publicar, Curtir, Compartilhar, Comentar, têm um significado especial se vistas sociologicamente: elas apontam para práticas de sociabilidade em rede, que são constituídas de formas de comunicação on-line, que levam à formação, à manutenção e à dinâmica de relações entre os agentes (i.e., páginas institucionais) e sua rede de conexões (i.e., seguidores) no campo (i.e., RSO) [Sterne 2003] [Santos e Cypriano 2014]. As RSO oferecem novas formas de acumulação desse tipo de capital, bem como o acesso a recursos que não estão facilmente disponíveis no mundo real para manutenção das relações sociais [Recuero 2012]. 
Para Bourdieu, o capital acumulado a partir das conexões sociais que os agentes estabelecem em um determinado campo é denominado de Capital Social [Bourdieu 1986]. Instituições que estão presentes nas RSO estão cada vez mais interessadas em saber de que maneira o Capital Social acumulado nesses meios digitais pode refletir no acúmulo desse capital no mundo real.

O Capital Social é acumulado a partir das práticas de sociabilidade em rede, que se cristalizam por meio das trocas realizadas entre os agentes presentes no campo. Nesta pesquisa, agentes são Páginas Institucionais (fanpages) e o campo é a rede Facebook. O volume do capital social acumulado por um agente depende da sua capacidade de mobilizar sua rede de conexões, na qual são estimuladas trocas e o reconhecimento mútuo entre integrantes dessa rede de conexões [Bourdieu 1986].

Outros autores estudaram o Capital Social [Coleman 1988] [Putnam 2001] [Lin et al. 2001] [Law e Chang 2008], além de Bourdieu. Apesar de indicadores do acúmulo desse tipo de capital terem sido estudados, principalmente, a partir da estrutura da rede de conexões de cada agente, não há consenso em como o Capital Social pode ser medido. No intuito de contribuir para a superação dessa lacuna, o presente trabalho apresenta uma abordagem para a construção de um indicador e para o cômputo do Capital Social acumulado em Páginas Institucionais dentro do Facebook, a partir do número de interações sociais capturadas de cada página.

A coleta de dados é direcionada por uma estratégia que se apoia no arcabouço teórico de Bourdieu. Esses dados coletados são processados e utilizados na extração automática e na mensuração de interações sociais no formato de sequência de ações. As frequências de ocorrência das sequências são associadas com a capacidade de mobilização dos seguidores conectados a cada Página Institucional (agente).

Caracterizando as relações entre páginas e seguidores dentro de um sistema de pesos, que leva em conta o número de ações executadas por seguidores dessas páginas em cada interação social, é construído um indicador do Capital Social acumulado. Em seguida, esse indicador juntamente com o número de interações sociais capturadas, são utilizados para computar o volume de Capital Social acumulado em cada Página Institucional.

O objetivo geral deste trabalho é mensurar o Capital Social acumulado em Páginas Institucionais no Facebook, levando-se em conta o número de interações sociais. A principal contribuição desta pesquisa é a abordagem empregada tanto para a construção de um indicador de acúmulo de Capital Social quanto na utilização desse indicador, juntamente com o número de interações sociais capturadas, para computar o volume de Capital Social acumulado.

Os resultados obtidos estão alinhados com as teorias de Bourdieu no que se refere 1) à representação do sistema de trocas entre agentes e sua rede de conexões, 2) à identificação da capacidade (incapacidade) de mobilização da rede de conexões de uma agente, e, 3) à capacidade (incapacidade) de mobilização de um agente que independe do tamanho da sua rede de conexões, 4) uma grande rede de contatos não garante um grande volume de Capital Social acumulado, ou seja, um volume maior de Capital Social acumulado depende da capacidade (efetiva) de mobilização da rede de conexões do agente, e 5) o Capital Social depende de um esforço incessante de sociabilidade, entretanto, um 
grande número de interações sociais não provoca necessariamente maior acúmulo de Capital Social, pois depende da efetiva mobilização da rede de conexões.

É importante observar que os dados coletados se referem a informações públicas e não pertencem ao perfil individual de qualquer seguidor de qualquer uma das Páginas Institucionais estudadas. As informações coletadas são mantidas sem identificação do perfil do usuário que a produziu. Ressalta-se que são explorados padrões de comportamento coletivos e impessoais, que podem ser extraídos automaticamente a partir de dados relacionados com as práticas de sociabilidade em rede.

Este trabalho está organizado da seguinte forma: Seção 2 detalha a metodologia empregada; Seção 3 apresenta e discute os resultados obtidos; Seção 4 elenca trabalhos relacionados; e Seção 5 apresenta as considerações finais.

\section{Metodologia}

\subsection{Recorte da pesquisa}

Dentro de uma RSO, a ação de Publicar inicia uma interação social. Essa ação também é chamada de estímulo social [Skinner 1953]. Na Psicologia Social [Mechner 2008], cada publicação seguida da execução, ou não, de ações, pode ser compreendida como uma contingência comportamental, ou também, uma interação social [Gomes e Pimentel 2011] [Gomes e Pimentel 2014]. Na Sociologia Digital [Ignatow e Robinson 2017], as práticas de sociabilidade dos agentes (i.e., página institucional) e sua rede de conexões (i.e., seguidores) no campo (i.e., RSO) são cristalizadas a partir da execução de funcionalidades intrínsecas à rede. Na rede Facebook, logo após uma publicação, as ações de Comentar, Curtir e Compartilhar ficam disponíveis e podem ser executadas pelos usuários conectados ao provedor do estímulo social [Gomes e Pimentel 2014].

Dentro da rede Facebook, a partir da ação de Publicar ficam disponíveis para a execução as ações de Comentar, Curtir e Compartilhar. Além do compartilhamento para fora, a rede Facebook permite o compartilhamento de uma publicação para dentro da rede. A ação de compartilhar analisada nesta pesquisa restringe-se apenas ao compartilhamento para dentro do Facebook (compartilhamento interno). Para analisar as práticas de sociabilidade em rede, foi selecionada uma instituição pública de ensino profissional com 13 câmpus. Cada câmpus tem autonomia para gerir sua fanpage seguindo diretrizes estabelecidas institucionalmente, e ainda, pelo governo federal ${ }^{1}$. O público conectado nessas páginas são, em sua imensa maioria, estudantes, professores e demais servidores. Cada página é sempre o único provedor de estímulos sociais e o público pode interagir somente executando as ações de curtir, comentar e compartilhar.

\subsection{Identificação dos dados a serem capturados}

O arcabouço teórico de Bourdieu [Bourdieu 1981] [Bourdieu 1986] [Bourdieu 1991], que fundamenta as pesquisas na Sociologia Digital [Ignatow e Robinson 2017], foi utilizado no direcionamento dos esforços na etapa de identificação dos dados a serem capturados. As funcionalidades de interação e comunicação que apontam para as práticas de sociabilidade em rede, foram identificadas, para esta pesquisa, como sendo as ações de Publicar, Curtir, Comentar e Compartilhar.

\footnotetext{
${ }^{1}$ www.governodigital.gov.br/transformacao/compras/orientacoes/orientacoes-redes-sociais-1
} 


\subsection{Coleta, transformação e carregamento}

Na etapa de coleta, transformação e carregamento dos dados identificados anteriormente, foi empregado o método ETL (Extract/Transform/Load) [Kimball e Ross 2002]. O acesso aos dados é feito utilizando-se a API Facebook ${ }^{2}$ durante o mês de janeiro de 2018. Foram coletados dados de todas as publicações (interações sociais) desde a data de criação de cada página. Atualmente, a API Facebook bloqueia o acesso aos dados de fanpages. Para contornar esse problema, até a liberação do acesso, é utilizado Selenium ${ }^{3}$ na coleta.

Scripts na linguagem Python ${ }^{4}$ foram implementados para o processamento do método ETL e os dados foram armazenados em um banco de dados MySQL ${ }^{5}$. Foi construída uma base de dados para cada página dentro do MySQL. Os dados capturados foram transformados para a etapa de extração de sequências de ações. Esses dados transformados foram carregados em uma outra base de dados que contém valores não-numéricos. A manipulação exclusiva de valores não-numéricos é uma restrição imposta pelo algoritmo de extração de sequências.

\subsection{Extração e mensuração de sequências}

Uma implementação do algoritmo GSP (Generalized Sequential Pattern) [Agrawal e Srikant 1995] foi elaborada para o aprendizado automático das sequências das ações. Originalmente, o GSP não manipula valores numéricos e o número de sequências extraídas depende de um limiar (frequência mínima) arbitrado pelo usuário. Na implementação aqui realizada, suporte mínimo considerado para extração de sequências candidatas foi $\alpha \geq 0$, portanto, todas sequências que possam constar na base de dados foram extraídas. São geradas sequências de tamanho 1 até as de tamanho igual ao número de colunas da base, sem valores de atributos repetidos. Por fim, o número de ocorrências (frequência) de cada sequência é calculado.

\subsection{Validação}

A validação dos resultados é feita a partir do confronto dos valores das frequências de ocorrência das sequências aprendidas com contagens retornadas por consultas elaboradas em Linguagem de Consulta Estruturada - SQL ${ }^{6}$. Cada sequência extraída via GSP de tamanho 3 é mapeada em uma consulta SQL. Na Listagem 1 é apresentado o mapeamento da sequência $! K ! L ! S$ na sua respectiva consulta SQL.

SELECT COUNT $(*)$ FROM 'posts' WHERE status_type IS NOT NULL AND num_comments $=0$ AND num_likes $=0$

AND num_shares $=0$

\section{Listagem 1. consultas em SQL}

Nessas consultas, são realizadas contagens iguais a 0 ou diferentes de 0 de cada ação. Considerando que as ações analisadas são Comentar, Curtir e Compartilhar, dessa forma, são feitas contagens do número de comentários, do número de curtidas e do número de compartilhamentos iguais a 0 ou diferentes de 0 .

\footnotetext{
${ }^{2}$ https://developers.facebook.com.

${ }^{3}$ https://www.seleniumhq.org/

${ }^{4}$ https://www.python.org/

${ }^{5}$ https://www.mysql.com/

${ }^{6}$ https://www.oracle.com/br/database/technologies/appdev/sql.html
} 
Havendo coincidência entre a frequência de ocorrência de cada sequência, obtidas a partir do aprendizado via GSP, e a contagem retornada pelas consultas SQL, esses resultados são considerados válidos. Em suma, os resultados são válidos se houver coincidência de valores entre o que foi aprendido via GSP com o que foi consultado via SQL.

\subsection{Cômputo do Capital Social}

Com o suporte teórico de Bourdieu [Bourdieu 1986], a capacidade de mobilização de cada página foi analisada a partir das frequências de ocorrência de cada sequência. Essa capacidade de mobilização se dá a partir de trocas realizadas entre agente e seguidores da página. Nesta pesquisa, foi considerado que as trocas se cristalizam dentro do Facebook a partir da execução ou não das ações Curtir, Comentar e Compartilhar, portanto, as trocas estão retratadas nas sequências aprendidas. A efetividade da capacidade de mobilização, por sua vez, é retratada pela execução de uma ou mais ações, sendo assim, as sequências que contém ações executadas no seu corpo estão associadas a efetividade da capacidade de mobilização do agente Página Institucional, como estudado em trabalho anterior pelos autores desta pesquisa [Cunha e Gomes 2019].

Caracterizando as relações entre cada página e seus seguidores dentro de um sistema de pesos, que leva em conta o número de ações executadas pelos seguidores dessas páginas em cada interação social, é construído um indicador do Capital Social acumulado, a partir das sequências aprendidas que estão ligadas à efetiva capacidade de mobilização. O número de ações executadas em cada interação social (sequência aprendida) é utilizado como peso para caracterizar a força da ligação de cada página com seus seguidores [Granovetter 1977]. Em seguida, esse indicador juntamente com o número de interações sociais capturadas de cada página institucional, são utilizados para computar o volume de Capital Social acumulado.

\section{Resultados e Discussão}

\subsection{Identificação dos dados e aplicação do método ETL}

Como resultado das fases de identificação e coleta dos dados foram construídas 13 bases de dados, uma para cada página institucional (fanpage). Nessas bases foram armazenadas informações como nome da página, identificador da página, identificador da postagem, conteúdo da postagem, tipo de mídia publicada na mensagem, data e hora da publicação, número de comentários, número de curtidas, número de compartilhamentos (internos).

$K, L, S \quad K, L, ! S \quad K, ! L, S \quad K, ! L, ! S \quad ! K, ! L, ! S$

\section{Listagem 2. Exemplo de Base de Dados não-numérica}

$\mathrm{Na}$ transformação dos dados, valores numéricos foram transformados em valores não-numéricos. O número de comentários maior que 0 é representado por $K$ e valores iguais a 0 por $! K$. O número de curtidas maior que 0 é representado por $L$ e valores iguais a 0 por $! L$. O número de compartilhamentos maior que 0 é representado por $S$ e valores iguais a 0 por $! S$.

Os dados transformados foram carregados em outra base com valores nãonuméricos, ou seja, para cada base de dados coletados da RSO, foi criada uma (nova) base de dados não-numéricos. A partir de cada base de dados não-numéricos foi realizado o aprendizado de sequência de ações. Um exemplo de como os dados estão organizados nas bases não-numéricas é apresentado na Listagem 2. 
Tabela 1. Mensuração da Incapacidade e Capacidade de Mobilização

\begin{tabular}{|c|c|c|c|c|c|c|c|c|c|c|c|c|}
\hline 1 & 2 & 3 & 4 & 5 & 6 & 7 & 8 & 9 & 10 & 11 & 12 & 13 \\
\hline Câmp. & !K!L!S & !KL!S & K!L!S & !K!LS & LK!S & !KLS & K!LS & KLS & total & I. Mob. & C. Mob. & \# seg. \\
\hline TRD & 8 & 292 & 0 & 1 & 108 & 304 & 0 & 325 & 1038 & $0,77 \%$ & $99,23 \%$ & 4967 \\
\hline CAT & 11 & 143 & 0 & 2 & 32 & 143 & 0 & 129 & 460 & $2,39 \%$ & $97,61 \%$ & 2921 \\
\hline IPO & 19 & 125 & 1 & 3 & 24 & 205 & 0 & 145 & 552 & $3,44 \%$ & $96,56 \%$ & 4086 \\
\hline $\mathrm{POI}$ & 0 & 46 & 0 & 0 & 2 & 75 & 0 & 23 & 146 & $0,00 \%$ & $100,00 \%$ & 764 \\
\hline URT & 54 & 314 & 1 & 10 & 76 & 394 & 0 & 219 & 1068 & $5,06 \%$ & $94,94 \%$ & 9638 \\
\hline CRI & 12 & 126 & 0 & 0 & 15 & 131 & 0 & 72 & 356 & $3,37 \%$ & $96,63 \%$ & 1586 \\
\hline CER & 71 & 471 & 2 & 20 & 116 & 410 & 1 & 312 & 1403 & $5,06 \%$ & $94,94 \%$ & 2905 \\
\hline HID & 4 & 100 & 0 & 0 & 25 & 82 & 0 & 44 & 255 & $1,57 \%$ & $98,43 \%$ & 1982 \\
\hline CPB & 23 & 427 & 2 & 3 & 68 & 349 & 0 & 190 & 1062 & $2,17 \%$ & $97,83 \%$ & 3679 \\
\hline PSS & 5 & 178 & 1 & 2 & 35 & 91 & 0 & 57 & 369 & $1,36 \%$ & $98,64 \%$ & 2836 \\
\hline RVD & 7 & 43 & 0 & 0 & 6 & 18 & 0 & 7 & 81 & $8,64 \%$ & $91,36 \%$ & 10293 \\
\hline IPA & 11 & 3 & 0 & 9 & 1 & 24 & 1 & 2 & 51 & $21,57 \%$ & $78,43 \%$ & 542 \\
\hline MOR & 546 & 210 & 0 & 0 & 52 & 331 & 0 & 217 & 1356 & $40,27 \%$ & $59,73 \%$ & 8819 \\
\hline
\end{tabular}

\subsection{Mensuração das sequências e da capacidade (incapacidade) de mobilização}

Exemplos das sequências de ações extraídas automaticamente de cada base de dados são apresentadas Listagem 3. Para o cômputo do Capital Social, foram consideradas apenas as sequências de ações de tamanho 3, pois essas sequências trazem no seu corpo uma associação entre a execução e/ou não das ações Comentar, Curtir e Compartilhar.

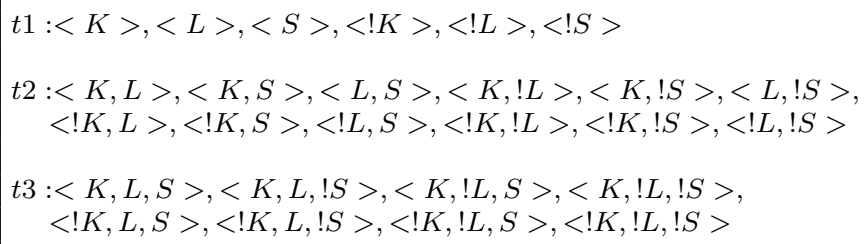

\section{Listagem 3. Exemplo de Sequências Candidatas}

Na Tabela 1 são apresentados uma identificação da Página Institucional de cada Câmpus (coluna 1), o número de ocorrência de cada sequência de ações de tamanho 3 em valores absolutos(colunas 2 a 9), a totalização dos valores de ocorrência dessas sequências (coluna 10), o indicador da incapacidade de mobilização (coluna 11), o indicador da capacidade de mobilização (complementar a incapacidade de mobilização - coluna 12), número de seguidores de cada página (tamanho da rede de conexões - coluna 13).

É necessário ressaltar que somatório do número de ocorrências de todas as sequências de tamanho 3, para um determinado Câmpus, corresponde ao número de linhas da base de dados desse Câmpus, ou seja, na mensuração das sequências de tamanho 3, foram escaneadas todas as ocorrências de sequências de ações armazenadas na base de dados que contém o registro das interações sociais ocorridas dentro da página do Câmpus. Dessa forma, para cada Câmpus (página institucional), o somatório do número de ocorrência das sequências $! K ! L ! S, ! K L ! S, K ! L ! S, ! K ! L S, L K ! S, ! K L S, K ! L S, K L S$ correspondem ao número de ocorrências das interações sociais armazenadas em cada base de dados.

\subsection{Validação dos Resultados}

Na Tabela 2 é apresentado o resultado da validação dos valores obtidos para a Página Institucional Câmpus TRD. Na segunda linha estão os números de ocorrência obtidos via GSP, e na terceira linha, os resultados das contagens feitas via consulta SQL. Para cada pagina foi construída uma tabela de validação tal como a Tabela 2. Os resultados 
Tabela 2. Exemplo de Validação de Resultados.

\begin{tabular}{|c||c|c|c|c|c|c|c|c|c|}
\hline Câmpus TRD & !K!L!S & !KL!S & K!L!S & !K!LS & LK!S & !KLS & K!LS & KLS & total \\
\hline GSP & 8 & 292 & 0 & 1 & 108 & 304 & 0 & 325 & 1038 \\
\hline SQL & 8 & 292 & 0 & 1 & 108 & 304 & 0 & 325 & 1038 \\
\hline$\neq$ & 0 & 0 & 0 & 0 & 0 & 0 & 0 & 0 & 0 \\
\hline
\end{tabular}

foram considerados válidos, pois o algoritmo GSP aprendeu a partir de todos os dados constantes em cada base (nenhum dado ficou de fora).

A validação dos resultados apresentados na Tabela 1 foi realizada a partir do confronto das frequências de ocorrências, obtidas a partir do aprendizado via GSP, com contagens obtidas a partir de consultas em SQL feitas em cada uma das 13 bases de dados. Os valores da Tabela 3 foram obtidos a partir da Tabela 1, portanto, validados os resultados da Tabela 1, os resultados apresentados na Tabela 3 também são considerados válidos.

\subsection{Cômputo do Capital Social}

Tabela 3. Indicador e Mensuração do Capital Social Acumulado

\begin{tabular}{|c|c|c|c|c|c|c|c|c|c|}
\hline 1 & 2 & 3 & 4 & 5 & 6 & 7 & 8 & 9 & 10 \\
\hline Câmpus & 0 açao & 1 açao & 2 acões & 3 ações & C. Mob. & Ind. CS & Int. Soc. & CS acum. & \# seg. \\
\hline TRD & $0,77 \%$ & $28,23 \%$ & $39,69 \%$ & $31,31 \%$ & $99,23 \%$ & $33,59 \%$ & 1038 & 348,66667 & 4967 \\
\hline CAT & $2,39 \%$ & $31,52 \%$ & $38,04 \%$ & $28,04 \%$ & $97,61 \%$ & $31,96 \%$ & 460 & 147 & 2921 \\
\hline IPO & $3,44 \%$ & $23,37 \%$ & $41,49 \%$ & $26,27 \%$ & $91,12 \%$ & $30,86 \%$ & 552 & 170,33333 & 4086 \\
\hline POI & $0,00 \%$ & $31,51 \%$ & $52,74 \%$ & $15,75 \%$ & $100,00 \%$ & $30,71 \%$ & 146 & 44,833333 & 764 \\
\hline URT & $5,06 \%$ & $30,43 \%$ & $44,01 \%$ & $20,51 \%$ & $94,94 \%$ & $29,99 \%$ & 1068 & 320,33333 & 9638 \\
\hline CRI & $3,37 \%$ & $35,39 \%$ & $41,01 \%$ & $20,22 \%$ & $96,63 \%$ & $29,68 \%$ & 356 & 105,66667 & 1586 \\
\hline CER & $5,06 \%$ & $35,14 \%$ & $37,56 \%$ & $22,24 \%$ & $94,94 \%$ & $29,50 \%$ & 1403 & 413,83333 & 2905 \\
\hline CPB & $2,17 \%$ & $40,68 \%$ & $39,27 \%$ & $17,89 \%$ & $97,83 \%$ & $28,81 \%$ & 1062 & 306 & 3679 \\
\hline PSS & $1,36 \%$ & $49,05 \%$ & $34,15 \%$ & $15,45 \%$ & $98,64 \%$ & $27,28 \%$ & 369 & 100,66667 & 2836 \\
\hline RVD & $8,64 \%$ & $53,09 \%$ & $29,63 \%$ & $8,64 \%$ & $91,36 \%$ & $23,05 \%$ & 81 & 18,666667 & 10293 \\
\hline IPA & $21,57 \%$ & $23,53 \%$ & $50,98 \%$ & $3,92 \%$ & $78,43 \%$ & $22,88 \%$ & 51 & 11,666667 & 542 \\
\hline MOR & $40,27 \%$ & $15,49 \%$ & $28,24 \%$ & $16,00 \%$ & $59,73 \%$ & $20,00 \%$ & 1356 & 271,16667 & 8819 \\
\hline
\end{tabular}

Na Tabela 3 são apresentados uma identificação da página institucional de cada Câmpus (coluna 1)e o percentual de sequências com 0 ações executadas (colunas 2), correspondente ao valor percentual da coluna 2 da Tabela 1 (sequência $! K ! L ! S$ ). Na coluna 3 , é apresentado o percentual de sequências com 1 ação executada, que corresponde ao percentual calculado a partir da soma das frequências de ocorrência das sequências $! K L ! S$, $K ! L ! S$ e $! K ! L S$.

Na coluna 4 da Tabela 3, é apresentado o percentual de sequências com 2 ações executadas, que corresponde ao percentual calculado a partir da soma das frequências de ocorrência das sequências $L K ! S, ! K L S$ e $K ! L S$. Na coluna 5 , é apresentado o percentual de sequências com 3 ações executadas, que corresponde ao percentual calculado a partir da frequência de ocorrência da sequência $K L S$ (coluna 9 da Tabela 1).

Seguindo na Tabela 3, na coluna 6 é apresentado o indicador da capacidade de mobilização (mesmos valores da coluna 12 da Tabela 1). Na coluna 7 é apresentado o indicador do Capital Social acumulado. Na coluna 8 é apresentado o número de interações sociais capturadas de cada página (mesmos valores da coluna 10 da Tabela 1). Na coluna 9 é apresentado o volume de Capital Social acumulado a partir do número de interações sociais capturadas de cada página. Na coluna 10 é apresentado o número de seguidores de cada página (tamanho da rede de conexões), valores da coluna 13 da Tabela 1. 
O indicador do Capital Social apresentado na coluna 7 foi obtido a partir da média ponderada das colunas 3 , 4 e 5 , de modo que o percentual das sequências com 1 ação executada recebeu peso 1, o percentual das sequências com 2 ações executadas recebeu peso 2 e o percentual das sequências com 3 ações executadas recebeu peso 3. Esses pesos são atribuídos para refletir a força das ligações entre seguidores e agente página institucional. Sendo assim, as ligações mais fracas têm peso 1 e as ligações mais fortes tem peso 3. O Capital Social acumulado apresentado na coluna 9 foi obtido pela multiplicação do indicador do Capital Social (coluna 7) pelo número de interações sociais (coluna 8).

\subsection{Discussão: mensuração das sequências e capacidade de mobilização}

Na presente pesquisa, as práticas de sociabilidade em rede são caracterizadas pela execução ou da não execução de ações Comentar, Curtir, Compartilhar logo após uma publicação. Essas práticas são representadas no formato de sequências de ações de tamanho 3, cujas frequências de ocorrência são apresentadas na Tabela 2, colunas de 2 a 9 .

Segundo Bourdieu, a mobilização de uma rede de contatos se dá a partir de trocas [Bourdieu 1986]. As trocas, dentro de uma RSO, são realizadas a partir de funcionalidades que viabilizam a execução de ações como Publicar, Comentar, Curtir, Compartilhar [Santos e Cypriano 2014]. Agentes com capacidade de mobilização são capazes de promover a realização de trocas, a partir de uma publicação, nas quais seus seguidores executam ações subsequentes. Em contraposição, a incapacidade de mobilização está ligada com a não execução das ações subsequentes a uma publicação, ou seja, o agente publica conteúdo que não mobiliza seus seguidores.

Observando-se a Tabela 1, a incapacidade de mobilização pode ser compreendida a partir da sequência $! K ! L ! S$, que expressa que o agente publicou um conteúdo e que 0 ações foram executadas em seguida. Na coluna 11, se destacam os valores $21,57 \%$ e 40,27\%, respectivamente, para as páginas institucionais dos Câmpus IPA e MOR, indicando que essas fanpages tem as mais altas incapacidades de mobilização (portanto, a mais baixa capacidade de mobilização) dentre os 13 agentes analisados. Observando-se o número de interações sociais, o Câmpus IPA tem o mais baixo número de interações sociais dentre os agentes estudados. Já o Câmpus MOR tem o segundo maior número de interações sociais. Esses resultados mostram que não basta prover um número grande de interações sociais para garantir uma alta capacidade de mobilização.

Além disso, Bourdieu destaca que a capacidade de mobilização de um agente independe do tamanho de sua rede de conexões [Santos e Cypriano 2014]. Continuando na Tabela 1, o número de seguidores de cada página é apresentado na coluna 13. Analisando o número de seguidores do Câmpus TRD, com maior capacidade de mobilização (menor incapacidade) e o Câmpus MOR com menor capacidade de mobilização, é possível observar que o Câmpus MOR tem quase o dobro de seguidores do Câmpus TRD.

Portanto, os resultados apresentados na Tabela 1 estão alinhados com as teorias de Bourdieu no que se refere 1) à representação do sistema de trocas entre agentes e sua rede de conexões, 2) à identificação da capacidade (incapacidade) de mobilização da rede de conexões de uma agente, e, 3) à capacidade (incapacidade) de mobilização de um agente que independe do tamanho da sua rede de conexões. 


\subsection{Discussão: Cômputo do Capital Social}

Na perspectiva de Bourdieu, o volume de Capital Social de um agente depende da rede de conexões que ele pode efetivamente mobilizar, portanto, a capacidade de mobilização é crucial para determinar o volume de Capital Social [Bourdieu 1986].

Na Tabela 3, a capacidade de mobilização está associada aos valores das colunas 3 a 5. Considerando as ações Curtir, Comentar e Compartilhar, dependendo da força da ligação entre agente e seguidores, o agente é capaz de estimular trocas a partir da execução de 1 ação até 3 ações. Na presente pesquisa, esse número de ações executadas é utilizado como peso para caracterizar a força da ligação [Granovetter 1977].

É admitido, portanto, que o Capital Social acumulado a partir de ligações fortes tenham peso maior no cômputo do volume de Capital Social do que o Capital Social acumulado por ligações fracas. Como mencionado anteriormente, o indicador do Capital Social apresentado na coluna 7 foi obtido a partir da média ponderada das colunas 3,4 e 5 da Tabela 3. Ao se propor um indicador do acúmulo de Capital Social a partir dessa média, o indicador promove um desbalanceamento em favor das ligações mais fortes entre agente e seus seguidores.

Observando-se a capacidade de mobilização, os percentuais das ações executadas e o indicador de acúmulo de Capital Social (colunas de 3 até 7 na Tabela 3), o Câmpus POI tem 100\% de capacidade de mobilização e o Câmpus TRD tem 99,23\%. Quando são observados os percentuais de ações executadas, o percentual da execução de 3 ações (ligação mais forte) é maior para o Câmpus TRD, com isso, o indicador do acúmulo de Capital Social do Câmpus TRD (33, 59\%) é maior que o do Câmpus POI (30, 71\%).

Bourdieu destaca que a manutenção e o crescimento do Capital Social depende de um esforço incessante de sociabilidade, trabalho contínuo de trocas no qual o reconhecimento é afirmado e reafirmado. No caso das RSO, esse esforço é percebido pelo número de publicações que a página institucional se dispõe a fazer. Cada publicação dá origem a uma interação social, portanto, a reprodução do Capital Social depende do esforço constante de publicar conteúdo que provoca a efetiva mobilização da rede de conexões.

O Capital Social acumulado, apresentado na coluna 9 na Tabela 3, foi obtido pela multiplicação do indicador do Capital Social (coluna 7) pelo número de interações sociais (coluna 8). Destaca-se a página institucional do Câmpus CER com maior número de interações sociais e maior acúmulo de Capital Social.

No entanto, um maior número de interações sociais não provoca necessariamente maior acúmulo de Capital Social, pois depende da efetiva mobilização da rede de conexões. Observando-se os valores das colunas 7, 8 e 9 da Tabela 3 para o Câmpus MOR, é possível notar que, mesmo com o segundo maior número de interações sociais, essa página institucional tem o menor valor para o indicador de Capital Social acumulado, o que afeta o volume de Capital Social acumulado.

Por fim, analisando os valores das colunas 7, 8 e 9 com a coluna 10 na Tabela 3, é possível perceber que uma rede de conexões ampla não garante necessariamente um grande acúmulo de Capital Social. O Câmpus RVD tem o maior número de seguidores, no entanto, o terceiro menor indicador do Capital Social e o segundo menor número de interações sociais. Sob a perspectiva de Bourdieu, o Capital Social acumulado nessa 
página pode aumentar, se o agente RVD investir esforços em provocar mais interações sociais que mobilizem efetivamente sua rede de contatos.

Sendo assim, os resultados apresentados na Tabela 3 estão alinhados com as teorias de Bourdieu no que se refere 4) uma grande rede de contatos não garante um grande volume de Capital Social acumulado, ou seja, um volume maior de Capital Social acumulado depende da capacidade (efetiva) de mobilização da rede de conexões do agente, e 5) o Capital Social depende de um esforço incessante de sociabilidade, entretanto, um grande número de interações sociais não provoca necessariamente maior acúmulo de Capital Social, pois depende da efetiva mobilização da rede de conexões.

Os resultados aqui apresentados limitam-se a realizar o cômputo do Capital Social acumulado em páginas de instituições de ensino dentro Facebook. Essas páginas têm a característica peculiar de que o único agente provedor de estímulos para as interações sociais é a instituição. Espera-se que abordagem aqui apresentada possa ser generalizada para qualquer agente, que seja único provedor de estímulos sociais, dentro de qualquer RSO. Em trabalhos futuros, o estudo aqui apresentado será ampliado para abarcar, por exemplo, o cômputo de Capital Social acumulado por "influenciadores digitais".

\section{Trabalhos Relacionados}

Os estudos sobre a inferência do Capital Social de agentes presentes nas Redes Sociais Online têm evoluído gradativamente, de abordagens que analisam da estrutura da rede como um grafo, para abordagens que analisam a atividade dos usuários (práticas de sociabilidade) dentro das RSO.

Na perspectiva de análise da estrutura da RSO para inferir o Capital Social, em [Venkatanathan et al. 2012] é apresentado um estudo sobre a relação entre a estrutura da rede Facebook, o Capital Social e como essa relação é moderada por traços de personalidade dos agentes. Em [Liu et al. 2014] é demonstrado como as informações estruturais implícitas das RSO podem fornecer informações importantes sobre a personalidade e o Capital Social dos usuários conectados nessas redes.

Em alinhamento com as conclusões obtidas nesses trabalhos, a pesquisa aqui apresentada destaca que agentes com maior volume de Capital Social acumulado são capazes de mobilizar uma rede de conexões mais ampla. Além disso, avança-se na compreensão de que o acúmulo do Capital Social depende das trocas, seguidas de reconhecimento mútuo, que são cristalizadas pelas funcionalidades disponíveis dentro da RSO.

Algoritmos de aprendizado de máquina voltados para tarefas preditivas, que empregam como técnica de aprendizado a regressão linear, são utilizados no estudo [Schams et al. 2018] para investigar a hipótese de que o Capital Social acumulado por usuários do Twitter e do Facebook, pode ser extraído de propriedades de rede e da atividade de cada usuário dentro da rede. Nesse trabalho, os usuários são ranqueados a partir de uma pontuação que é inferida automaticamente pelos algoritmos de classificação.

Avançando a partir da análise do trabalho de Schamns, nesta pesquisa são objeto de análise os comportamentos coletivos dos usuários que fazem parte da rede de conexões de uma Página Institucional (agente). O Capital Social acumulado por um agente é computado a partir da utilização de um algoritmo aprendizado de máquina voltado para tarefas descritivas, que emprega como técnica de aprendizado a associação, para extrair 
sequências de ações relacionadas às práticas de sociabilidade em rede dos seguidores de uma página. O Capital Social acumulado de cada agente é calculado a partir a mensuração das sequências de ações executadas por seguidores das páginas.

Em relação a esses trabalhos, a presente pesquisa traz, como contribuições, a extração automática de sequências de ações que caracterizam as práticas de sociabilidade em rede dentro das RSO, e ainda, o cômputo do Capital Social acumulado dentro de Páginas Institucionais no Facebook, a partir da mensuração das sequências de ações. Deve ser destacado que, nesta pesquisa, o volume de Capital Social acumulado é computado a partir das interações sociais capturadas (atividade dos usuários) de cada página.

\section{Considerações Finais}

Neste trabalho foi apresentada uma abordagem para computar o Capital Social acumulado em Páginas Institucionais no Facebook, levando-se em conta o número de interações sociais que ocorrem dentro dessas páginas. O arcabouço teórico de Bourdieu foi utilizado no direcionamento da coleta de dados relativos às práticas de sociabilidade em rede, e ainda, no estabelecimento de um indicador do Capital Social acumulado e do volume de Capital Social acumulado.

Os resultados aqui apresentados estão alinhados com as teorias de Bourdieu no que se refere 1) à representação do sistema de trocas entre agentes e sua rede de conexões, 2) à identificação da capacidade (incapacidade) de mobilização da rede de conexões de uma agente, e, 3) à capacidade (incapacidade) de mobilização de um agente que independe do tamanho da sua rede de conexões, 4) uma grande rede de contatos não garante um grande volume de Capital Social acumulado, ou seja, um volume maior de Capital Social acumulado depende da capacidade (efetiva) de mobilização da rede de conexões do agente, e 5) o Capital Social depende de um esforço incessante de sociabilidade, entretanto, um grande número de interações sociais não provoca necessariamente maior acúmulo de Capital Social, pois depende da efetiva mobilização da rede de conexões.

O escopo da pesquisa aqui apresentada está limitado a análise das ações executadas dentro de Páginas Institucionais por seguidores dessas páginas. O campo considerado limita-se a rede social Facebook. Apesar disso, deseja-se que a abordagem empregada para computar o Capital Social apresentada nesta pesquisa, possa ser generalizada para ser aplicada a qualquer página ou perfil institucional presente em qualquer RSO.

\section{Referências}

Agrawal, R. e Srikant, R. (1995). Mining sequential patterns. In Proc. of the Inter. Conf. on Data Engineering (ICDE '95), pages 3-14. IEEE Computer Society.

Bourdieu, P. (1981). Men and machines. In Advances in Social Theory and Methodology, pages 304-317. Routledge \& Kegan Paul, Boston.

Bourdieu, P. (1986). The forms of capital. In Handbook of Theory and Research for the Sociology of Education, pages 241-258. Greenwood Press, New York.

Bourdieu, P. (1991). Language and Symbolic Power. Social theory. John B. Thompson Ed., Polity Press.

Coleman, J. S. (1988). Social capital in the creation of human capital. American journal of sociology, 94:S95-S120. 
Cunha, K. M. R. e Gomes, A. K. (2019). Measurement of mobilization capacity via actions sequence learning within institutional facebook fan pages. In Proc. of the 25th Braz. Symp. on Mult. and the Web, WebMedia 2019, pages 317-320. ACM.

Gomes, A. K. e Pimentel, M. d. G. C. (2011). A technique for human-readable representation e evaluation of media-based social interactions in social networks. In Proc. of the 17th Braz. Symp. on Mult. and the Web, WebMedia 2011, pages 119-126. SBC.

Gomes, A. K. e Pimentel, M. d. G. C. (2014). Evaluation of Media-Based Social Interactions: Linking Collective Actions to Media Types, Applications, and Devices in Social Networks, pages 75-95. Springer Vienna, Vienna.

Granovetter, M. S. (1977). The strength of weak ties. In Social networks, pages 347-367. Elsevier.

Ignatow, G. e Robinson, L. (2017). Pierre bourdieu: theorizing the digital. Information, Communication \& Society, 20(7):950-966.

Kimball, R. e Ross, M. (2002). The Data Warehouse Toolkit: The Complete Guide to Dimensional Modeling. John Wiley \& Sons, Inc., New York, NY, USA, 2nd edition.

Law, S. P.-M. e Chang, M. K. (2008). Fostering knowledge exchange in online communities: a social capital building approach. International Conference on Information Systems, ICIS 2008 Proceedings, page 173.

Lin, N., Cook, K. S., e Burt, R. S. (2001). Social capital: Theory and research. Transaction Publishers.

Liu, Y., Venkatanathan, J., Goncalves, J., Karapanos, E., e Kostakos, V. (2014). Modeling what friendship patterns on facebook reveal about personality and social capital. ACM Trans. Comput.-Hum. Interact., 21(3):17:1-17:20.

Mechner, F. (2008). Behavioral contingency analysis. Behavioral Processes, 78(2):124144.

Putnam, R. (2001). Social capital: Measurement and consequences. Canadian journal of policy research, 2(1):41-51.

Recuero, R. (2012). O capital social em rede: Como as redes sociais na internet estão gerando novas formas de capital social. Contemporânea, 10(3):597-617.

Santos, F. C. e Cypriano, C. P. (2011). Blogs e wikis: duas formas de colaboração em redes sociais. Revista Ciência em Movimento - Rede Metodista, 13:07 - 19. https://www.metodista.br/revistas/revistas-ipa/index.php/ EDH/article/view/100/64.

Santos, F. C. e Cypriano, C. P. (2014). Redes sociais, redes de sociabilidade. Revista Brasileira de Ciências Sociais, 29:63-78.

Schams, S., Hauffa, J., e Groh, G. (2018). Analyzing a user's contributive social capital based on acitivities in online social networks and media. In Comp. Proc. of the The Web Conference 2018, WWW'18, pages 1457-1464.

Skinner, B. F. (1953). Science and Human Behavior. New York: Macmillan.

Sterne, J. (2003). Bourdieu, technique and technology. Cultural Studies, 17:367-389.

Venkatanathan, J., Karapanos, E., e Gonçalves, J. (2012). Network, personality and social capital. In Proc. of Annual Web Science Conf., WebSci' 12, pages 326-329. ACM. 